\title{
Explicit Exact Solutions to Some One Dimensional Conformable Time Fractional Equations
}

\author{
Alper Korkmaz * \\ Department of Mathematics, Çankırı Karatekin University, Çankırı 18200, Turkey
}

December 16, 2016

\begin{abstract}
The exact solutions of some conformable time fractional PDEs are presented explicitly. The modified Kudryashov method is applied to construct the solutions to the conformable time fractional Regularized Long WaveBurgers (RLW-Burgers, potential Korteweg-de Vries (KdV) and clannish random walker's parabolic (CRWP) equations. Initially, the predicted solution in the finite series of a rational form of an exponential function is substituted to the ODE generated from the conformable time fractional PDE by using wave transformation. The coefficients used in the finite series are determined by solving the algebraic system derived from the coefficients of the powers of the predicted solution.
\end{abstract}

Keywords: modified Kudryashov method; conformable time fractional RLW-Burgers Equation; conformable time fractional potential KdV Equation; conformable time fractional CRWP equation; conformable derivative

MSC2010: 35C07;35R11;35Q53.

PACS: 02.30.Jr; 02.70.Wz; 04.20.Jb

\section{Introduction}

The last several decades witness giant development on symbolic programming. As a result of these developments, many effective techniques have been derived to

*korkmazalper@yandex.com 
solve numerous problems. The power of solving complicated algebraic computations more rapidly than human directs many researcher to develop, improve and implement new methods on the computer based platforms. Thus, the solution techniques for the PDEs have also been moved to the computers to gain time. Having no general methods to be used for all nonlinear PDEs attracts the researcher to derive new methods to solve them. Due to few of them integrable, the predicted solution techniques are more common to solve nonlinear PDEs. A predicted solution is substituted into the solution and the parameters are determined by algebraic ways in this class of the solution methods. Giving a solution to a PDE does not require the method to work for another one. This aspect forces the researchers to try the same method for the solutions of the other PDEs.

The simple equation, first integral, ansatz and various types of expansion methods are some of the recent efficient techniques that are used to solve nonlinear PDEs. The developments on the fractional derivatives change the focus of the applied mathematicians to solve fractional type differential equations. Some look for the ways to implement the techniques to the fractional forms of the nonlinear PDEs. The key is the existence and validity of the change rule in addition to necessary other properties while solving this family of nonlinear PDEs. The fundamental properties of the conformable fractional derivatives enable the compulsory conditions to implement these methods to fractional nonlinear partial derivatives despite some constraints.

Here, the modified form of the Kudryashov method is applied to construct the solution of some conformable time fractional nonlinear PDEs. Before starting the implementation of the method to generate the solutions, some fundamentals and properties of the conformable derivative are stated below.

\section{Conformable Fractional Derivative}

Let $\alpha$ be in $(0,1]$. Then, the conformable derivative is given as

$$
D^{\alpha}(u(t))=\lim _{\tau \rightarrow 0} \frac{u\left(t+\tau t^{1-\alpha}\right)-u(t)}{\tau}
$$

for a conformable differentiable function $u:[0, \infty) \rightarrow \mathbb{R}$ in the half space $t>0[1]$. The conformable derivative has significant properties given below. Assuming the derivative order $\alpha \in(0,1]$, and supposing that $v=v(t)$ and $w=w(t)$ are $\alpha$ differentiable for all $t>0$, the conformable derivative satisfies:

- $D^{\alpha}(a v+b w)=a D^{\alpha}(v)+b D^{\alpha}(w)$

- $D^{\alpha}\left(t^{p}\right)=p t^{p-\alpha}, \forall p \in \mathbb{R}$ 
- $D^{\alpha}(\lambda)=0$, for all constant function $v(t)=\lambda$

- $D^{\alpha}(v w)=v D^{\alpha}(w)+w D^{\alpha}(v)$

- $D^{\alpha}\left(\frac{v}{w}\right)=\frac{w D^{\alpha}(v)-v D^{\alpha}(w)}{w^{2}}$

- $D^{\alpha}(v)(t)=t^{1-\alpha} \frac{d v}{d t}$

for $\forall a, b \in \mathbb{R}[2$, 3].

Some significant properties such as the Taylor series expansion, Laplace transform, exponential function and the chain rule are defined in [4]. The following theorem completes the required conditions for the implementation of the Kudryashov method in the modified form to the fractional PDEs in conformable sense.

Theorem 1 Let $v$ be an $\alpha$-differentiable function in conformable sense and differentiable and suppose that $w$ is also differentiable and defined in the range of $v$. Then,

$$
D^{\alpha}(v \circ w)(t)=t^{1-\alpha} w^{\prime}(t) v^{\prime}(w(t))
$$

\section{The Modified Kudryashov Method}

Consider a nonlinear PDE of the form

$$
F\left(v, v_{t}^{\alpha}, v_{x}, v_{t}^{2 \alpha}, v_{x x}, \ldots\right)=0
$$

where $v=v(x, t)$ and $\alpha \in(0,1]$ stands for the order of the conformable derivative. The wave transformation

$$
v(x, t)=v(\xi), \xi=x-\frac{c}{\alpha} t^{\alpha}
$$

reduces the dimension of the (3) and generates and ordinary differential equation of the form

$$
G\left(v, v^{\prime}, v^{\prime \prime}, \ldots\right)=0
$$

where the prime $\left({ }^{\prime}\right)$ stands for the derivative of $v$ with respect to $\xi$. The variants of this transformation is used in the works [5, 6].

Let

$$
v(\xi)=\sum_{i=0}^{n} a_{i} P^{n}(\xi)
$$

be a solution to (5) where all $a_{i}, 0 \leq i \leq n$ are constants satisfying $a_{n} \neq 0$ and

$$
P(\xi)=\frac{1}{1+d A^{\xi}}
$$


is a solution to the ODE

$$
P^{\prime}(\xi)=P(\xi)(P(\xi)-1) \ln A
$$

where $d$ and $A$ are nonzero constants with $A>0$ and $A \neq 1$. The key in the method is to determine $n$ by balancing the highest order derivative and the nonlinear terms in (5). Thus, the solution (6) is formed with unknown coefficients $a_{i}$. Substituted it into (5) and rearranging the resultant equation with respect to the powers of $P(\xi)$ gives an algebraic system. Solving this algebraic system for $a_{i}, 0 \leq i \leq n$ explicitly gives the relations between the parameters of the solutions $a_{i}, 0 \leq i \leq n$ and the remaining parameters derived from structure of the equation or the other factors.

\section{The Conformable Fractional RLW-Burgers Equa- tion}

Consider the time fractional RLW-Burgers equation of the form for $v=v(x, t)$

$$
D_{t}^{\alpha} v+p v_{x}+q v v_{x}+r v_{x x}+s v_{x x t}=0, t>0, x \in \mathbb{R}
$$

where $D_{t}^{\alpha}$ is the fractional derivative operator in conformable sense, $p, q, r$, and $s$ are constant parameters. The integer ordered form of the equation appears in the work [7] to describe surface water waves propagation in a channel. Some basic properties covering existence, uniqueness and boundedness of the solutions of some problems related to RLW-Burgers are expressed in that study. Zhao and Xuan [8] examine in details the existence and convergence properties of solutions of the RLW-Burgers equation. The monotone and oscillatory kink type waves are discussed in details by Zhou and Liu[9]. Some trigonometric and hyperbolic type exact solutions are derived by using some expansion methods [10] as some complex solutions are constructed by direct algebraic method[11]. $\left(1 / G^{\prime}\right)$-expansion method is also capable of traveling wave solutions to the RLW-Burgers equation[12]. The wave transform (4) reduces the RLW-Burgers equation (8) to

$$
(-c+p) v+\frac{q}{2} v^{2}+r v^{\prime}-c s v^{\prime \prime}-K=0
$$

where ' denotes the derivative with respect to the wave variable $\xi$ and $K$ is integration constant. Balancing $v^{2}$ and $v^{\prime \prime}$ gives $n=2$. Thus, the predicted solution should be in the form $v(\xi)=a_{0}+a_{1} P(\xi)+a_{2} P^{2}(\xi)$. Substituting this solution 
into the (9) and using (7) yield

$$
\begin{aligned}
& \left(-6(\ln (A))^{2} c s a_{2}+1 / 2 q a_{2}^{2}\right) P^{4}(\xi) \\
& +\left(-2 c s a_{1}(\ln (A))^{2}+10(\ln (A))^{2} c s a_{2}+2 \ln (A) r a_{2}+q a_{1} a_{2}\right) P^{3}(\xi) \\
& +\left(q a_{0} a_{2}+\ln (A) r a_{1}-4(\ln (A))^{2} c s a_{2}-2 \ln (A) r a_{2}+3 c s a_{1}(\ln (A))^{2}+\frac{1}{2} q a_{1}^{2}+(p-c) a_{2}\right) P^{2}(\xi) \\
& +\left(-c s a_{1}(\ln (A))^{2}-\ln (A) r a_{1}+q a_{0} a_{1}-c a_{1}+p a_{1}\right) P(\xi)-c a_{0}+p a_{0}+1 / 2 q a_{0}^{2}-K=0
\end{aligned}
$$

When the coefficients of the powers of $P(\xi)$ are equated to zero, the system of algebraic equations

$$
\begin{array}{r}
-c a_{0}+p a_{0}+1 / 2 q a_{0}{ }^{2}-K=0 \\
-c s a_{1}(\ln (A))^{2}-\ln (A) r a_{1}+q a_{0} a_{1}-c a_{1}+p a_{1}=0 \\
q a_{0} a_{2}+\ln (A) r a_{1}-4(\ln (A))^{2} c s a_{2}-2 \ln (A) r a_{2}+3 c s a_{1}(\ln (A))^{2}+1 / 2 q a_{1}{ }^{2}-c a_{2}+p a_{2}=0 \\
-2 c s a_{1}(\ln (A))^{2}+10(\ln (A))^{2} c s a_{2}+2 \ln (A) r a_{2}+q a_{1} a_{2}=0 \\
-6(\ln (A))^{2} c s a_{2}+1 / 2 q a_{2}{ }^{2}=0
\end{array}
$$

is obtained. The solution of this system for $a_{0}, a_{1}, a_{2}, c, K$ gives two different solution set for $a_{0} \neq 0$. The first one

$$
\begin{aligned}
a_{0} & =\frac{1}{5} \frac{6(\ln (A))^{2} r s-5 p \ln (A) s-r}{\ln (A) q s} \\
a_{1} & =0 \\
a_{2} & =-\frac{12 \ln (A) r}{5 q} \\
c & =-\frac{1}{5} \frac{r}{\ln (A) s} \\
K & =\frac{1}{50} \frac{\left(6(\ln (A))^{2} r s-5 p \ln (A) s-r\right)\left(6(\ln (A))^{2} r s+5 p \ln (A) s+r\right)}{q(\ln (A))^{2} s^{2}}
\end{aligned}
$$

makes the solution to $(9)$ as

$$
v(\xi)=\frac{6(\ln (A))^{2} r s-5 p \ln (A) s-r}{5 \ln (A) q s}-\frac{12 \ln (A) r}{5 q\left(1+d A^{\xi}\right)^{2}}
$$

where $q s \neq 0$. Thus, the solution of the RLW-Burgers equation (8) is expressed explicitly as

$$
v_{1}(\xi)=\frac{6(\ln (A))^{2} r s-5 p \ln (A) s-r}{5 \ln (A) q s}-\frac{12 \ln (A) r}{5 q\left(1+d A^{x+\frac{1}{5} \frac{r}{\ln (A) s} \frac{t^{\alpha}}{\alpha}}\right)^{2}}
$$


The second solution to (11) for $a_{0}, a_{1}, a_{2}, c, K$ is of the form

$$
\begin{aligned}
a_{0} & =-\frac{1}{5} \frac{-6(\ln (A))^{2} r s+5 p \ln (A) s-r}{\ln (A) q s} \\
a_{1} & =-\frac{24 \ln (A) r}{5 q} \\
a_{2} & =\frac{12 \ln (A) r}{5 q} \\
c & =\frac{1}{5} \frac{r}{\ln (A) s} \\
K & =\frac{1}{50} \frac{\left(6(\ln (A))^{2} r s-5 p \ln (A) s+r\right)\left(6(\ln (A))^{2} r s+5 p \ln (A) s-r\right)}{q(\ln (A))^{2} s^{2}}
\end{aligned}
$$

Thus, the solution is obtained as

$$
v(\xi)=-\frac{-6(\ln (A))^{2} r s+5 p \ln (A) s-r}{5 \ln (A) q s}-\frac{24 \ln (A) r}{5 q\left(1+d A^{\xi}\right)}+\frac{12 \ln (A) r}{5 q\left(1+d A^{\xi}\right)^{2}}
$$

where $q s \neq 0$. Using the original variables, the solution to the RLW-Burgers equation in conformable fractional form (8) is written as

$$
\begin{aligned}
v_{2}(x, t)= & -\frac{-6(\ln (A))^{2} r s+5 p \ln (A) s-r}{5 \ln (A) q s}-\frac{24 \ln (A) r}{5 q\left(1+d A^{x-\frac{1}{5} \frac{r}{\ln (A) s}} \frac{t^{\alpha}}{\alpha}\right)} \\
+ & \frac{12 \ln (A) r}{5 q\left(1+d A^{x-\frac{1}{5} \frac{r}{\ln (A) s}} \frac{t^{\alpha}}{\alpha}\right)^{2}}
\end{aligned}
$$

\section{The Conformable Fractional Potential KdV Equa- tion}

In this section, the conformable time fractional potential $\mathrm{KdV}$ equation of the form

$$
D_{t}^{\alpha} v+p v_{x}^{2}+q v_{x x x}=0
$$

is solved by using the modified Kudryashov method. The wave transformation (4) reduces (18) to

$$
-c v^{\prime}+p\left(v^{\prime}\right)^{2}+q v^{\prime \prime \prime}=0
$$


where' denotes the derivative with respect to the wave variable $\xi$. The balance between $\left(v^{\prime}\right)^{2}$ and $v^{\prime \prime \prime}$ gives the degree of the predicted solution as $n=2$. Thus, the predicted solution $v(\xi)=a_{0}+a_{1} P(\xi)$ is substituted to the 19 to give

$$
\begin{aligned}
& \left(6(\ln (A))^{3} q a_{1}+(\ln (A))^{2} p a_{1}^{2}\right) P^{4}(\xi)+\left(-12(\ln (A))^{3} q a_{1}-2(\ln (A))^{2} p a_{1}^{2}\right) P^{3}(\xi) \\
& +\left(7(\ln (A))^{3} q a_{1}+(\ln (A))^{2} p a_{1}^{2}-\ln (A) c a_{1}\right) P^{2}(\xi)+\left(-(\ln (A))^{3} q a_{1}+\ln (A) c a_{1}\right) P(\xi)=0
\end{aligned}
$$

When the coefficients of each power of $P(\xi)$ to zero, the algebraic system of equations

$$
\begin{aligned}
-(\ln (A))^{3} q a_{1}+\ln (A) c a_{1} & =0 \\
7(\ln (A))^{3} q a_{1}+(\ln (A))^{2} p a_{1}{ }^{2}-\ln (A) c a_{1} & =0 \\
-12(\ln (A))^{3} q a_{1}-2(\ln (A))^{2} p a_{1}{ }^{2} & =0 \\
6(\ln (A))^{3} q a_{1}+(\ln (A))^{2} p a_{1}{ }^{2} & =0
\end{aligned}
$$

The solution set of this system for $\left\{a_{0}, a_{1}, c\right\}$ gives

$$
\begin{aligned}
a_{1} & =-6 \frac{\ln (A) q}{p} \\
c & =(\ln (A))^{2} q
\end{aligned}
$$

for arbitrary constant $a_{0}$. The solution of (19) is constructed as

$$
v(\xi)=a_{0}-6 \frac{\ln (A) q}{p} \frac{1}{1+d A^{\xi}}
$$

where $p \neq 0$ and $a_{0}$ is an arbitrary constant. Thus, the solution of the conformable fractional potential $\mathrm{KdV}(18)$ takes the form

$$
v(x, t)=a_{0}-6 \frac{\ln (A) q}{p} \frac{1}{1+d A^{x-(\ln (A))^{2} q \frac{t^{\alpha}}{\alpha}}}
$$

with a nonzero $q$.

\section{The Conformable Fractional CRWP Equation}

Consider the conformable time fractional CRWP equation given in the form

$$
D_{t}^{\alpha} v+p v_{x}+q v v_{x}+r v_{x x}=0
$$

This equation models the motion of two populations interacting each other but wishing to live separately for $\alpha=1[13$, 14]. Some exact solutions of the CRWP 
equation including some trigonometric and hyperbolic functions besides Jacobi elliptic functions are derived in those studies. Afterwards, the Jumarie time fractional form of the equation is solved exactly by using the Kudryashov method[15]. The wave transformation (4) reduces the CRWP equation to

$$
-(c-p) v^{\prime}+\frac{q}{2}\left(v^{2}\right)^{\prime}+r v^{\prime \prime}=0
$$

Integration the last equation once gives

$$
\left.-(c-p) v+\frac{q}{2} v^{2}\right)+r v^{\prime}=K
$$

where $K$ is constant of integration. The balance between the nonlinear and the highest order derivative terms occurs for $n=1$. The predicted solution is constructed as $a_{0}+a_{1} P(\xi), a_{1} \neq 0$. Substituting this solution and its derivative to (refcrwpode) gives

$$
\left(\frac{1}{2} q a_{1}^{2}+r a_{1} \ln (A)\right) P^{2}(\xi)+\left(-r a_{1} \ln (A)+q a_{0} a_{1}-c a_{1}+p a_{1}\right) P(\xi)+\frac{1}{2} q a_{0}^{2}-c a_{0}+p a_{0}-K=0
$$

This system requires

$$
\begin{aligned}
\frac{1}{2} q a_{0}{ }^{2}-c a_{0}+p a_{0}-K & =0 \\
-r a_{1} \ln (A)+q a_{0} a_{1}-c a_{1}+p a_{1} & =0 \\
\frac{1}{2} q a_{1}^{2}+r a_{1} \ln (A) & =0
\end{aligned}
$$

The solution sets for this algebraic equation system can be determined as

$$
\begin{aligned}
a_{0} & =\frac{r \ln (A)+\sqrt{r^{2}(\ln (A))^{2}-2 q K}}{q} \\
a_{1} & =-2 \frac{r \ln (A)}{q} \\
c & =\sqrt{r^{2}(\ln (A))^{2}-2 q K}+p
\end{aligned}
$$

and

$$
\begin{aligned}
a_{0} & =-\frac{-r \ln (A)+\sqrt{r^{2}(\ln (A))^{2}-2 q K}}{q} \\
a_{1} & =-2 \frac{r \ln (A)}{q} \\
c & =-\sqrt{r^{2}(\ln (A))^{2}-2 q K}+p
\end{aligned}
$$


Thus, the solutions generated from these two sets are constructed as

$$
\begin{aligned}
& v_{4}(\xi)=\frac{r \ln (A)+\sqrt{r^{2}(\ln (A))^{2}-2 q K}}{q}-2 \frac{r \ln (A)}{q} \frac{1}{1+d A^{\xi}} \\
& v_{5}(\xi)=-\frac{-r \ln (A)+\sqrt{r^{2}(\ln (A))^{2}-2 q K}}{q}-2 \frac{r \ln (A)}{q} \frac{1}{1+d A^{\xi}}
\end{aligned}
$$

Finally, the solutions of the conformable fractional CRWP equation 25 are formed as

$$
\begin{aligned}
& v_{4}(x, t)=\frac{r \ln (A)+\sqrt{r^{2}(\ln (A))^{2}-2 q K}}{q}-2 \frac{r \ln (A)}{q} \frac{1}{1+d A^{x-\left(\sqrt{r^{2}(\ln (A))^{2}-2 q K}+p\right)} \frac{t^{\alpha}}{\alpha}} \\
& v_{5}(x, t)=-\frac{-r \ln (A)+\sqrt{r^{2}(\ln (A))^{2}-2 q K}}{q}-2 \frac{r \ln (A)}{q} \frac{1}{1+d A^{x+\left(\sqrt{r^{2}(\ln (A))^{2}-2 q K}+p\right)} \frac{t^{\alpha}}{\alpha}}
\end{aligned}
$$

for nonzero $q$.

\section{Conclusion}

The explicit exact solutions to some time fractional nonlinear PDEs in conformable sense are constructed by using the modified Kudryashov method. The existence of chain rule for the conformable derivative gives opportunity to reduce the related PDEs to compatible ODEs of integer orders. The predicted solution having the finite series of rational exponential function is substituted to the resultant ODE. Afterwards, the unknown parameters are determined by using fundamental algebra operations.

The solutions of conformable fractional RLW-Burgers, potential KdV and CRWP equations are obtained. The forms of the solution contains a finite series of powers of rational exponential functions. 


\section{References}

[1] Khalil, R., Al Horani, M., Yousef, A., \& Sababheh, M. (2014). A new definition of fractional derivative. Journal of Computational and Applied Mathematics, 264, 65-70.

[2] Atangana, A., Baleanu, D., \& Alsaedi, A. (2015). New properties of conformable derivative. Open Mathematics, 13(1), 1-10.

[3] Çenesiz, Y., Baleanu, D., Kurt, A., \& Tasbozan, O. (2016). New exact solutions of Burgers' type equations with conformable derivative. Waves in Random and Complex Media, 1-14.

[4] Abdeljawad, T. (2015). On conformable fractional calculus. Journal of computational and Applied Mathematics, 279, 57-66.

[5] Eslami, M., \& Rezazadeh, H. (2015). The first integral method for Wu-Zhang system with conformable time-fractional derivative. Calcolo, 1-11.

[6] Hosseini, K., Mayeli, P., \& Ansari, R. (2016). Modified Kudryashov method for solving the conformable time-fractional Klein-Gordon equations with quadratic and cubic nonlinearities. Optik-International Journal for Light and Electron Optics(corrected proof.).

[7] Bona, J. L., Pritchard, W. G., \& Scott, L. R. (1981). An evaluation of a model equation for water waves. Philosophical Transactions of the Royal Society of London A: Mathematical, Physical and Engineering Sciences, 302(1471), 457510.

[8] Zhao, H., \& Xuan, B. (1997). Existence and convergence of solutions for the generalized BBM-Burgers equations with dissipative term. Nonlinear Analysis: Theory, Methods \& Applications, 28(11), 1835-1849.

[9] Zhou, Y., \& Liu, Q. (2012, August). Kink waves and their evolution of the RLW-burgers equation. In Abstract and Applied Analysis (Vol. 2012), Article ID 109235, 1-14.

[10] İnan, İ. E., Uğurlu, Y., \& Kılıç, B. (2009). Traveling Wave Solutions of the RLW-Burgers Equation and Potential Kdv Equation by Using the-Expansion Method. Cankaya University Journal of Science and Engineering, 12(2), 103110.

[11] Bas, E., \& Kilic, B. (2010). New Complex Solutions for Rlw Burgers Equation, Generalized Zakharov-Kuznetsov Equation and Coupled Korteweg-De Vries Equation. World Applied Sciences Journal, 11(3), 256-262. 
[12] Yokuş, A. (2015). An expansion method for finding traveling wave solutions to nonlinear pdes, Istanbul Ticaret Universitesi Fen Bilimleri Dergisi, 14, 27, 65-81.

[13] Uğurlu, Y., \& Kaya, D. (2007). Analytic method for solitary solutions of some partial differential equations. Physics Letters A, 370(3), 251-259.

[14] Kiliç, B. (2013). Exact Solutions for Nonlinear Evolution Equations with Jacobi Elliptic Function Rational Expansion Method. World Applied Sciences Journal, 23(12), 81-88.

[15] Bulut, H., \& Kilic, B. (2013). Exact solutions for some fractional nonlinear partial differential equations via Kudryashov method. E-Journal of New World Sciences Academy, 8(1), 24-31.

(C) 2016 by the authors; licensee Preprints, Basel, Switzerland. This article is an open access article distributed under the terms and conditions of the Creative Commons Attribution (CC BY) license http://creativecommons.org/licenses/by/4.0/). 